\title{
Impacts of Atomically Flat Si (111) Surfaces on Novel Photonic Crystal Designs
}

\author{
Alyssa Prasmusinto, Abdelrahman Al-Attili, Hideo Arimoto, Shinichi Saito \\ Electronics and Computer Science, Faculty of Physical Sciences and Engineering, University of Southampton \\ Southampton, United Kingdom SO17 1BJ
}

\begin{abstract}
Characteristics of photonic crystals are very sensitive to fabrication-induced disorders due to scattering losses. Here, we propose to use atomically flat silicon (111) surfaces, defined by anisotropic wet etching. We theoretically examined the impacts of the surfaces on the novel designs of photonic crystals.
\end{abstract}

\section{INTRODUCTION}

Photonic crystals (PhCs) provide the ability to control electromagnetic waves in the sub-wavelength regime through the generation of photonic band gaps (PBG) [1]. By using $\mathrm{PhCs}$, we can reduce the footprint of a photonic device, while minimizing the energy consumption down to the order of $\mathrm{fJ} / \mathrm{bit}$, offering the potential realization of all-optical switches and interconnections based on existing CMOS technologies [2]. However, fabrication of such nano-scale structures still remains a practical challenge, as their performance is sensitive to structural disturbances, such as line-edge roughness [3], [4]. As a consequence, it is difficult to fabricate $\mathrm{PhCs}$ with sufficient manufacturing tolerance ready for mass production.

In this paper, we propose a novel 2-dimensional $\mathrm{PhC}$ design using silicon (Si) (111) planes that can be fabricated through anisotropic wet etching. The Si (111) planes have a much slower etching rate in anisotropic wet etchants compared to other planes, thus capable of producing atomically flat interfaces with reduced roughness [5], [6]. By using Si (111) surfaces to construct PhCs, we can also expand the manufacturing tolerance against processing, since the etching is self-limited after defining the Si (111) surfaces [6]. Here, we study the impacts of Si (111) surfaces on the PBG generation and compare the results with conventional circular $\mathrm{PhCs}$. We also review the impact of the surfaces on the PhC's optical confinement capabilities.

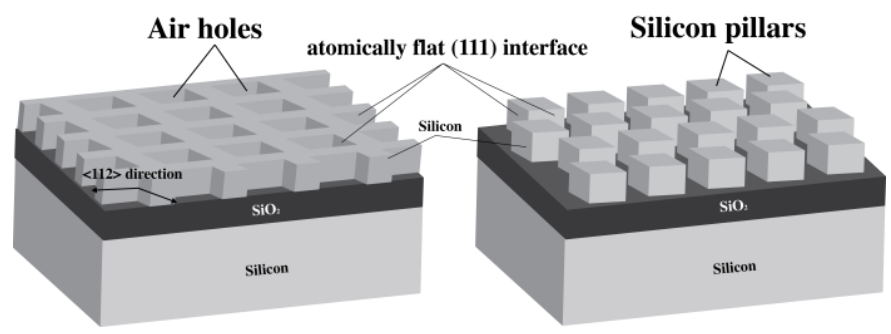

Fig. 1. Schematic of 2-dimensional PhCs with parallelogram lattice points on SOI. Examples of square lattice silicon rods (right) and air holes (left).

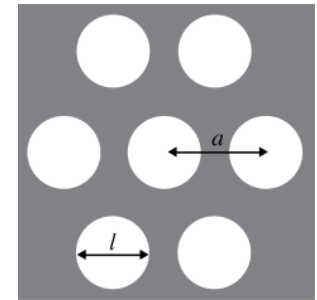

(a)

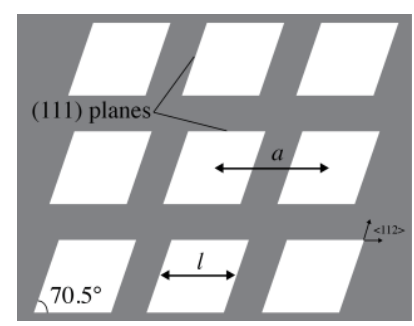

(c)

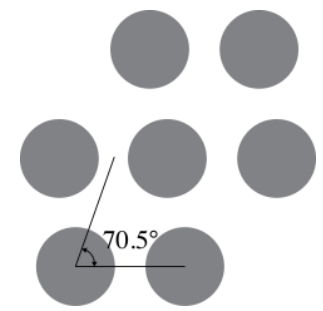

(b)

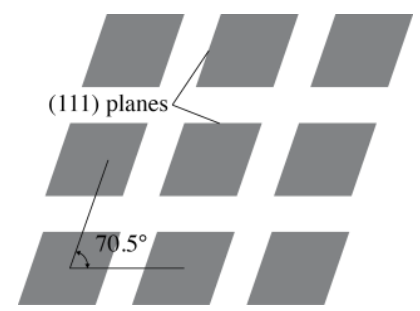

(d)
Fig. 2. Simulated photonic crystals. (a) Circular air holes (white) on silicon slab in triangular lattice, (b) circular silicon pillars (grey) on air in triangular lattice tilted by $70.5^{\circ}$, (c) parallelogram air holes on silicon slab in square lattice, and (d) parallelogram silicon rods on air in square lattice titled by $70.5^{\circ}$.

\section{Photonic CRYSTAL DESIGN}

We assume the Silicon-On-Insulator (SOI) substrate with the Si (110) surface on the top layer. If we employ anisotropic alkali wet etching, we can define two surfaces with atomically flat Si (111) interface perpendicular to the substrate (Fig. 1). One surface is parallel perpendicular to the $\langle 112\rangle$ direction, while the other surface is perpendicular along to the $\langle 110\rangle$ direction.

We propose a novel PhC that makes use of the these two Si (111) surfaces, forming parallelograms with interior angles $70.53^{\circ}$ and $109.47^{\circ}$ (Figs. 1 and 2). To simulate our $\mathrm{PhC}$, we consider a SOI wafer with the thickness of $300 \mathrm{~nm}$. We investigate the transverse electric (TE) band structure for air holes in SOI slab and the transverse magnetic (TM) polarization band structure for $\mathrm{Si}$ pillars for various lattice structures (Table I). For both PhCs, we examined a square and a triangular lattice. We also examined a tilting of the square lattice by $70.5^{\circ}$ to make the symmetry of the lattice the same as that of the parallelogram (Fig. 2 (d)) [7]. For completeness, we also simulated a tilted triangular lattice (Fig. 2b). As a 
TABLE I. OPTIMUM DESIGN PARAMETERS CHARACTERIZING THE PBG

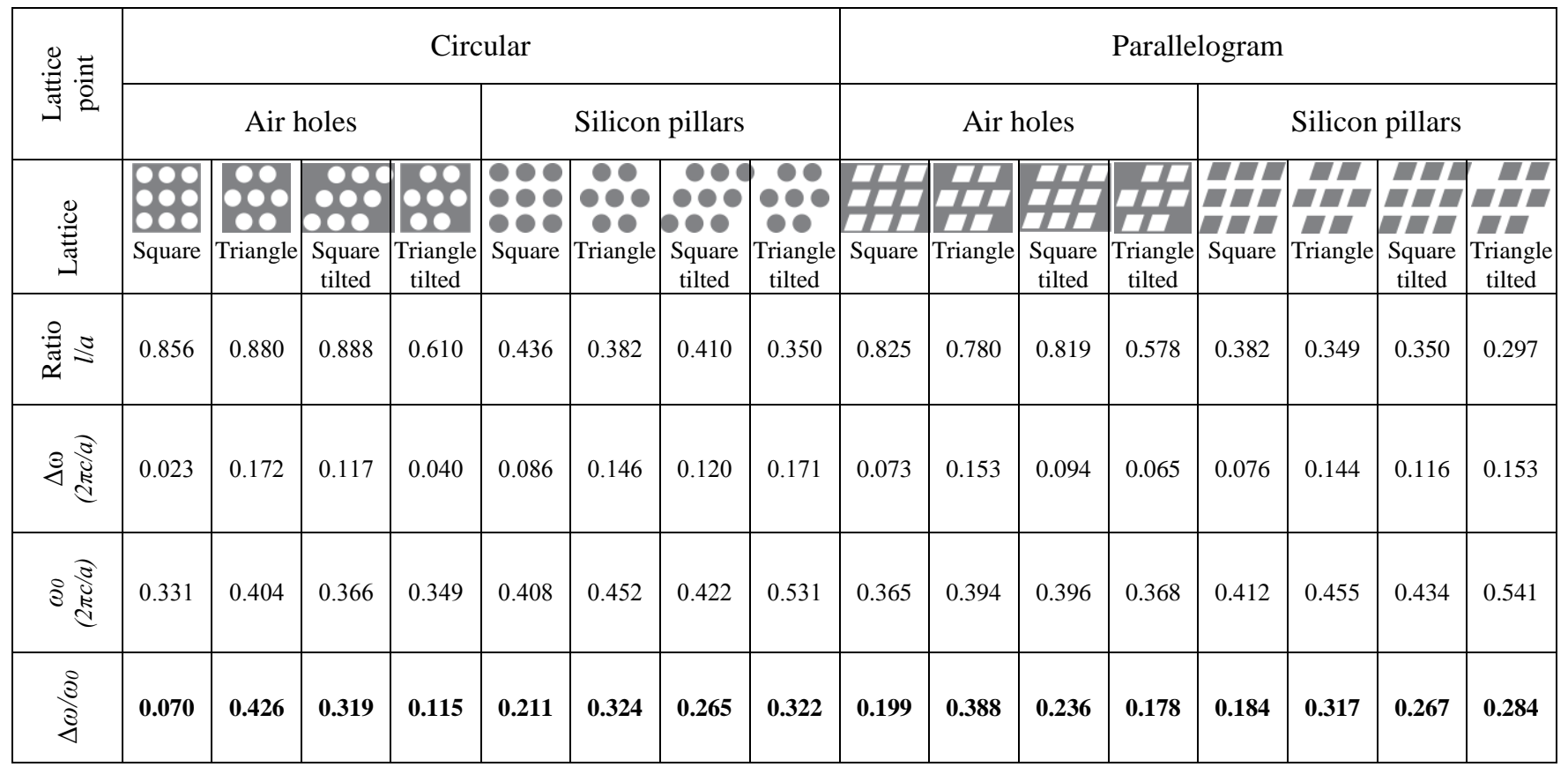

(a)
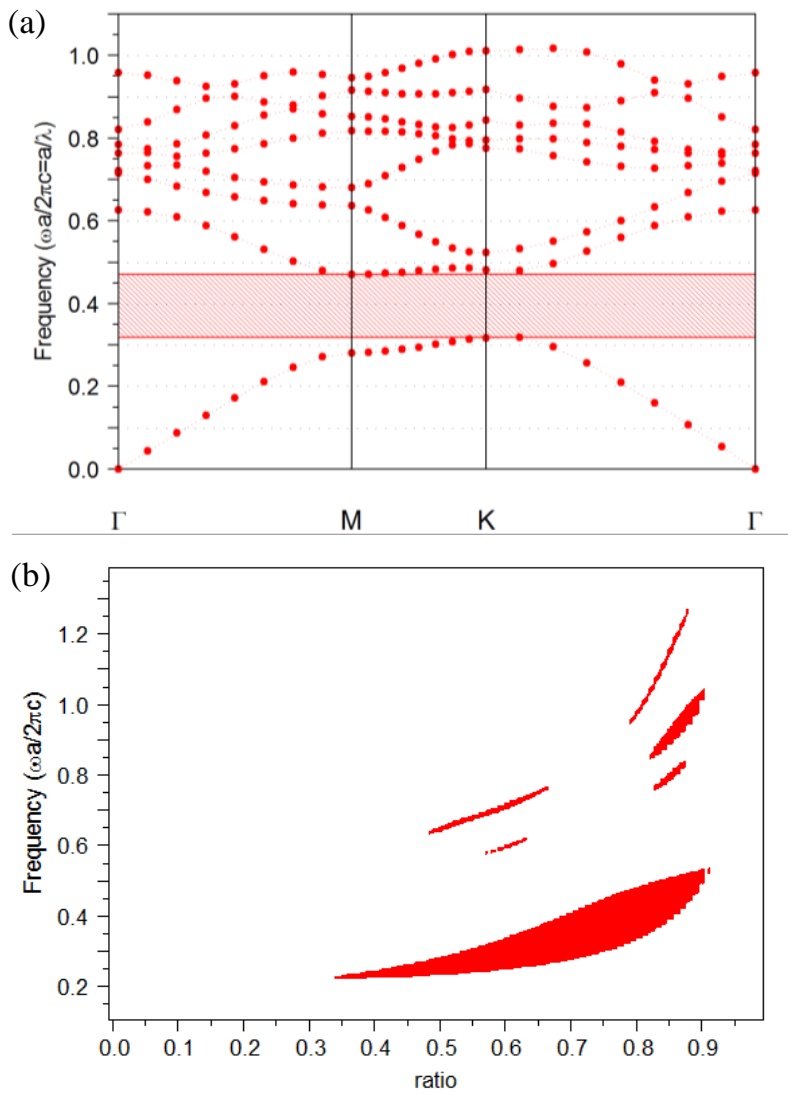

Fig. 3. Band structure (a) and gap map (b) of triangular lattice air holes PhCs with parallelogram lattice points. The shaded region in the band structure indicates a PBG. Red in the gap map indicates TE PBGs. reference, we simulated conventional $\mathrm{PhCs}$ with circular lattice points.

\section{BANDGAP SIMULATION RESUltS}

We simulated the PhCs using RSoft BandSOLVE with the plane wave expansion and characterized the PBG between the first and the second band. We simulated band structures for various filling fractions in each lattice structure. In a $\mathrm{PhC}$ with circular lattice points, the band structures are described by the ratio, $l / a$, where $l$ is the diameter and $a$ is the lattice constant (Fig. 2a). On the other hand, in a $\mathrm{PhC}$ with parallelogram lattice points, $l$ is defined by the width of the parallelogram (Fig. 2c). We obtained the optimum ratio to find out the largest PBG $(\Delta \omega)$ for each lattice structure. Fig. 4 compares the change in $\Delta \omega$ between all lattice configurations as the $l / a$ ratio is varied. We also obtained the frequency at the center of the gap, $\omega_{0}$. A summary of the results can be seen in Table I.

We then characterized the structures using the gap-tomidgap ratio, $\omega_{R}=\Delta \omega / \omega_{0}$. In the case of square lattices, tilting the lattice of the PhC strucutre by $70.5^{\circ}$ enlarges the $\Delta \omega$ and enhances $\omega_{R}$. This is expected from the parallelogram PhCs [7], but it is also true for circular lattice. In the case of triangular lattices, on the other hand, the tilting does not increase the $\omega_{R}$, presumably because the tilting angle does not agree with the triangular lattice. If we compare $\omega_{R}$ among lattice types, the triangular lattice produces the largest $\omega_{R}$, irrespective of whether the lattice points are made of holes or pillars for both circular and parallelogram lattice points [7]. 


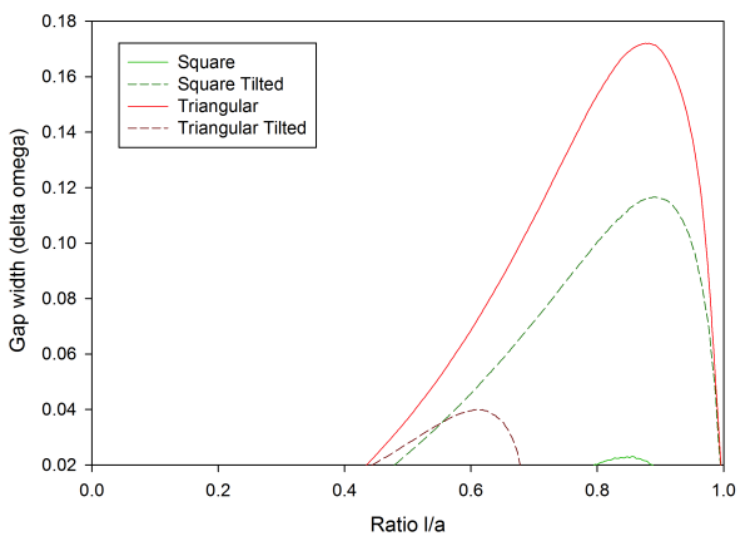

(a)

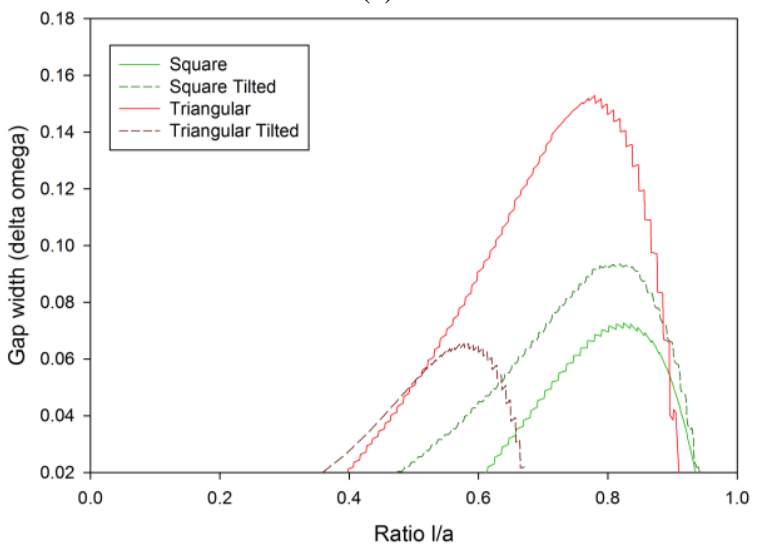

(c)

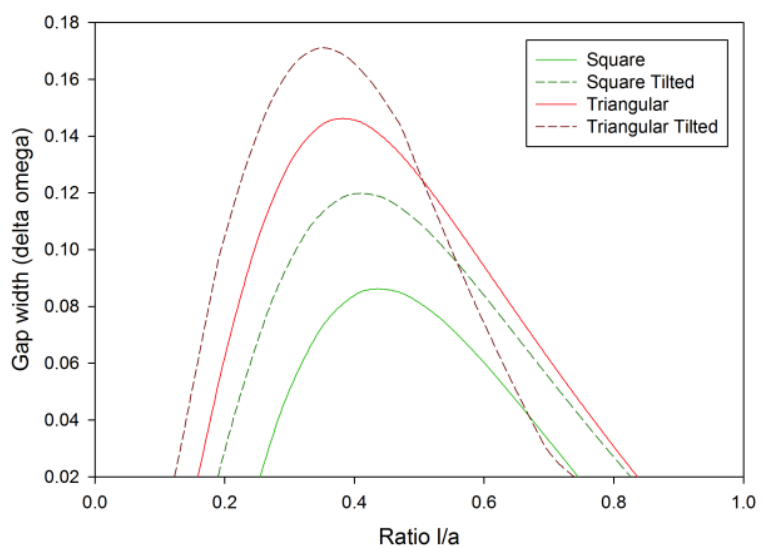

(b)

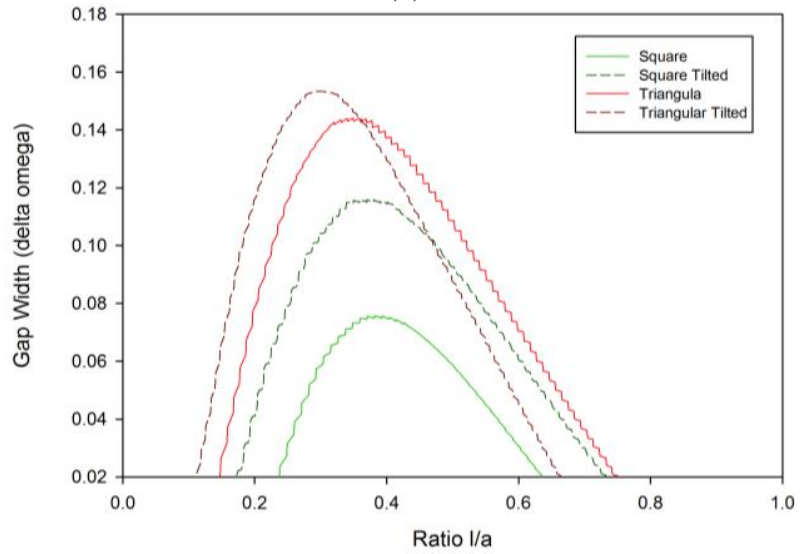

(d)

Fig. 4. Comparison of gap width versus ratio $l / a$ between various lattice arrangements with circular lattice points for (a) air holes and (b) silicon pillars and parallelogram lattice points for (c) air holes and (d) silicon pillars.

(a)

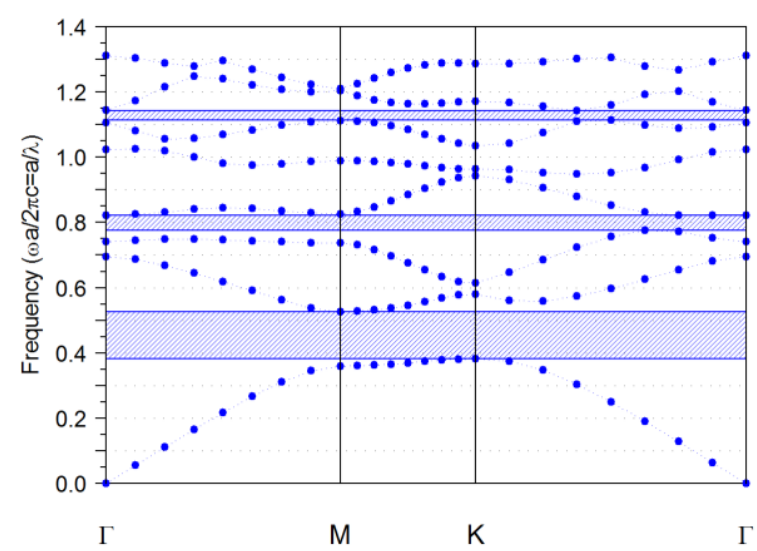

(b)

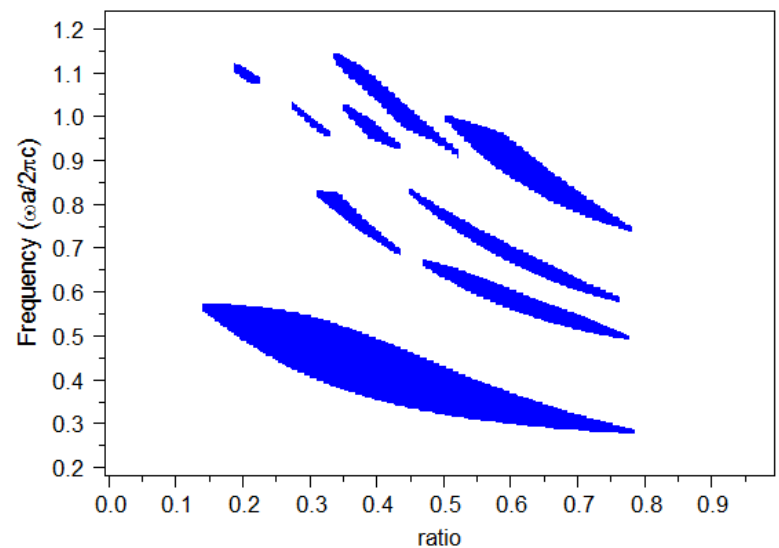

Fig. 5. Band structure (a) and gap map (b) of triangular lattice silicon pillars PhCs with parallelogram lattice points. The shaded region in the band structure indicates a PBG. Blue in the gap map indicates TM PBGs.

In the case of air holes, lattices with parallelogram lattice points produce comparable $\omega_{R}$ to those with circular lattice points. Square and tilted triangular lattices even show larger $\omega_{R}$. Among all air holes configurations, the largest $\omega_{\mathrm{R}}$ of 0.426 with a TE gap width of $0.172(2 \pi c / a)$ and a gap center at $0.404(2 \pi c / a)$ appears in the case of the triangular lattice with circular lattice points. The same lattice arrangement with parallelogram lattice points yields a $\omega_{R}$ of 0.388 with a TE gap width of $0.153(2 \pi c / a)$ and a gap center at $0.394(2 \pi c / a)$, which is the largest value among all lattices with parallelogram lattice points. 
Similar results were observed from the silicon pillars. The differences between $\omega_{R}$ between two lattice points are less significant compared to air-holes based lattices. Among the parallelogram lattice points configurations, the triangular lattice with parallelogram lattice points produces the highest $\omega_{R}$ of 0.317 with a TM gap of $0.144(2 \pi c / a)$ and a gap center at $0.455(2 \pi c / a)$. This does not differ much from the same lattice with circular lattice points, which yields a $\omega_{R}$ of 0.324 with a TM gap of $0.146(2 \pi c / a)$ and a gap center at $0.452(2 \pi c / a)$.

As shown above, band structures of $\mathrm{PhCs}$ with parallelograms are not drastically different from those of the conventional $\mathrm{PhCs}$, suggesting the possibility of integrating $\mathrm{Si}$ (111) surfaces into $\mathrm{PhCs}$ without compromising performance. It must also be noted that the simulations are made without taking into account the reduced sidewall roughness of the $\mathrm{PhCs}$ with parallelograms. We may therefore expect even more notable differences in $\omega_{R}$ upon the smoothing of the sidewalls.

\section{Cavity Simulation Results}

To demonstrate the optical confinement using our PhCs, we introduced a $\mathrm{H} 0$ cavity into the triangular air holes lattice [1]. The HO cavity is formed by shifting two adjacent lattice points in the center outward in the $x$-direction by a distance $d$ [8]. In our simulations, we fixed $d$ to $0.12 a$. The width of the parallelogram lattice points and the lattice constant were optimized to achieve a resonant wavelength of $1550 \mathrm{~nm}$ inside the cavity. We simulated the structure using RSoft FullWAVE with the finite-difference time-domain approach and computed the quality factor, $Q$, and the modal volume, $V$. We used the ratio, $Q / V$, which is proportional to the Purcell factor [1] to describe the strength of the optical confinement. As a reference, we also simulated the same cavity in the triangular lattice $\mathrm{PhC}$ with circular air holes, as summarized in Table II.

The lattice with the circular lattice points produced a $Q$ value of $\sim 21,000$, which is over five times larger than the value of the lattice with the parallelogram lattice points $(\sim 4,000)$. Based on the $Q / V$ values, the circle-based lattice seems to exhibit stronger optical confinement capabilities. Even though the $\omega_{R}$ of these two lattices only differ by 0.038 , the impacts on the $Q$ values were significant. This would be attributed from the field distributions as shown in Table II. For circular lattice points, the monopole inside the cavity of the lattice with circular lattice points was more symmetrically distributed compared with that of the parallelogram lattice points. Due to the slanted sides of the parallelogram, the confined field inside the lattice is slightly tilted. The asymmetric geometry of the cavity slightly deforms the monopole and responsible for the additional loss mechanisms.

\section{CONCLUSION}

We theoretically examined 2-dimensional $\mathrm{PhC}$ structures using lattices that can be defined by Si (111) planes. We have shown that structures with parallelogram lattice points will have gap-to-midgap ratios comparable with conventional
TABLE II. PARAMETERS CHARACTERIZING THE H0 CAVITY

\begin{tabular}{|c|c|c|c|}
\hline & Circular \\
\hline & & \\
\hline
\end{tabular}

circular lattice points. Among lattices with parallelogram lattice points, the highest gap-to-midgap ratio of 0.388 was obtained from the triangular air holes configuration. We have also shown that the $Q$ value of approximately 4,000 would be achievable. Therefore, the use of Si (111) planes for PhCs will be suitable for applications, which will not require the extremely high- $Q$ values. For example, we can apply these $\mathrm{PhCs}$ for the grating coupler with the atomically flat low loss waveguide [9].

\section{ACKNOWLEDGMENTS}

This work is supported by EPSRC standard grant (EP/M009416/1), EPSRC manufacturing fellowship (EP/M008975/1), EU FP7 Marie-Curie Carrier-IntegrationGrant (PCIG13-GA-2013-618116), and Hitachi. The raw data from this paper can be obtained from the University of Southampton ePrints research repository, DOI: $10.5258 /$ SOTON/383488.

\section{REFERENCES}

[1] E. Yablonovitch, J. Mod. Opt., vol. 41, no. 2, pp. 173-194, 1994.

[2] M. Notomi, Reports Prog. Phys., vol. 73, no. 9, p. 096501, 2010.

[3] Z. H. Zhu, W. M. Ye, J. R. Ji, X. D. Yuan, and C. Zen, Appl. Phys. B Lasers Opt., vol. 88, no. 2, pp. 231-236, 2007.

[4] Z. Jakšić, M. Maksimović, O. Jakšić, D. Vasiljević-Radović, Z. Djurić, and A. Vujanić, Microelectron. Eng., vol. 83, pp. 17921797, 2006.

[5] K. K. Lee, D. R. Lim, L. C. Kimerling, J. Shin, and F. Cerrina, Opt. Lett., vol. 26, no. 23, pp. 1888-1890, 2001.

[6] R. E. Oosterbroek, J. W. Berenschot, H. V. Jansen, A. J. Nijdam, G. Pandraud, a. Van Den Berg, and M. C. Elwenspoek, $J$. Microelectromechanical Syst., vol. 9, no. 3, pp. 390-398, 2000.

[7] R. Wang, X. H. Wang, B. Y. Gu, and G. Z. Yang, J. Appl. Phys. vol. 90, no. 9, pp. 4307-4313, 2001.

[8] Z. Zhang and M. Qiu, Opt. Express, vol. 12, no. 17, pp. 3988-3995, 2004.

[9] H. Arimoto, M. K. Husain, A. Prasmusinto, K. Debnath, A. AlAttili, R. Petra, H. M. H. Chong, G. T. Reed, and S. Saito (submitted to Group IV Photonics, 2015). 
